\title{
SOLIDIFICATION ANALYSIS OF ALUMINUM-BASED MEDIUM ENTROPY CASTING ALLOY
}

\author{
Jarosław PIĄTKOWSKI, Paweł GRADOŃ, Martyna LACHOWSKA \\ Silesian University of Technology, Faculty of Materials Engineering and Metallurgy, \\ Katowice, Silesia, Poland, EU, \\ jaroslaw.piatkowski@polsl.pl, pawel.gradon@polsl.pl, martyna.lachowska@polsl.pl
}

https://doi.org/10.37904/metal.2019.744

\begin{abstract}
Alloys with increased configurational entropy are new group of materials with very broad range of possible properties, but their application potential is currently limited because of complicated and inefficient manufacturing processes used mainly on laboratory scale. Paper presents the results of preliminary research aimed on manufacturing castings made of medium entropy alloys (MEA) by industry scale casting technologies. The main aim of the presented research was to determine the influence of melting and casting conditions on the solidification process of selected Al-based MEA's. The alloys were poured into sample moulds made of material with heat transfer coefficient similar to those used in industrial scale manufacturing processes. Furthermore, the pattern used in the mould preparation was designed to create zones of varied heat dissipation rates and thus solidification rate. Solidification process was characterized by use of thermalderivative analysis and differential scanning calorimetry. The structure in as-cast state and chemical composition in micro-areas were investigated using SEM microscopy and EDS analysis.
\end{abstract}

Keywords: Metallurgy, casting, aluminum alloys, medium entropy alloys, solidification, thermal analysis

\section{INTRODUCTION}

High entropy alloys (HEA) and medium entropy alloys (MEA) [1], also known as multi-principal element alloys (MPEA) [2], are a new class of metal alloys comprised of more than four principal alloying elements. This is the fundamental difference between HEAs and conventional alloys which contain only one or two principal elements and is connected to one of four core effects observed in these entropic alloys [3,4], the so called "cocktail effect" [5] with tree other effects being: sluggish diffusion, severe lattice distortion and high entropy.

The cocktail effect leads to staggering amount of possible combinations of properties in multi component alloys as base characteristics of alloying elements like, for example, low density of alloy composed of light elements, are added to properties resulting from numerous potential synergies between them [4,5].

Beside the properties derived directly from the composition, the other core effects extend the potential of HEAs even further. For example sluggish diffusion leads to exceptional high-temperature resistance and stability [6] and severe lattice distortion is used to explain high strength of BCC HEAs [7] and hardness of FCC HEAs [8].

High entropy effect is the namesake of these new materials, and long thought to be their main characteristic. It was proposed that high configurational entropy in alloying system consisting of five or more selected elements in equiatomic composition can lead to formation of only one simple solid solution phase by minimisation of its Gibbs energy relative to e.g. intermetallic phases, even in spite of Gibbs phase rule $[1,9,10]$.

The first papers concerning these new materials were published circa 2004 and since then the topic of HEA became very widely recognized and has been still actively developed.

Early classification of MPEAs (Figure 1) is based on the value of configurational entropy of ideal solid solution with equimolar composition which is calculated using Boltzmann's principle by equation (1) [1].

According to this first classification, the HEA is an alloy of five or more elements mixed in proportions ranging between 35 and 5 at\% consisting mainly of simple solid solution phases in which the value of $\Delta S^{c o n f}$ is equal 
or higher than $1.61 \mathrm{R}$. In case of alloy with 2 to 4 principal elements the high configurational entropy effect is not the main stabilising force for solid solution phases ( $\Delta S^{\text {conf }}$ in range from $0.69 R$ to $1.39 R$ ), but other characteristics of HEAs are present and so this group of alloys was named medium entropy alloys (MEA) [11].

$\Delta S^{\text {conf }}=-k \ln w=-R \ln \frac{1}{n}=R \ln \mathrm{n}$,

where:

$k$ - the Boltzmann's constant,

$w$ - the number of ways of mixing,

$R$ - the universal gas constant,

$n$ - the number of major alloying elements.

Abovementioned classification evolved over the years to two definitions of MPEAs. One is based on configurational entropy value and the other on alloy composition [12].

The compositional definition states that HEAs are expected to consist of at least 5 major alloying elements in concentrations between 35 and 5 at $\%$ and can include other, minor elements in concentrations less than 5 at\%. $[10,13]$. On the other hand, the entropy definition is based only on ideal configurational entropy values

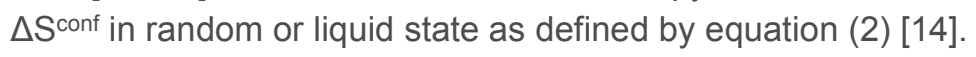

$\Delta S^{\text {conf }}=-R \sum_{i}^{n} x_{i} \ln x_{i}$,

where:

$n$ - the number of elements,

$x_{i}$ - the mole fraction of element $\mathrm{i}$.

This definition implies that HEAs must have $\Delta S^{\text {conf }}>1.5 R$. For MEAs the range is considered to be $1.0 R \leq$ $\Delta S^{\text {conf }} \leq 1.5 R$. Alloys with configurational entropy less than $1 R$ are called low entropy alloys (LEA) and they include traditional metal alloys which contain only one or two major alloying elements [10].

Graphical representation of both definitions has been shown in Figure 1 on the right hand scheme. It is also worth mentioning that, although the definitions overlap in most cases, there are examples of alloys that meet the requirements of only one definition and are often still considered either HEAs or MEAs. $[10,13]$
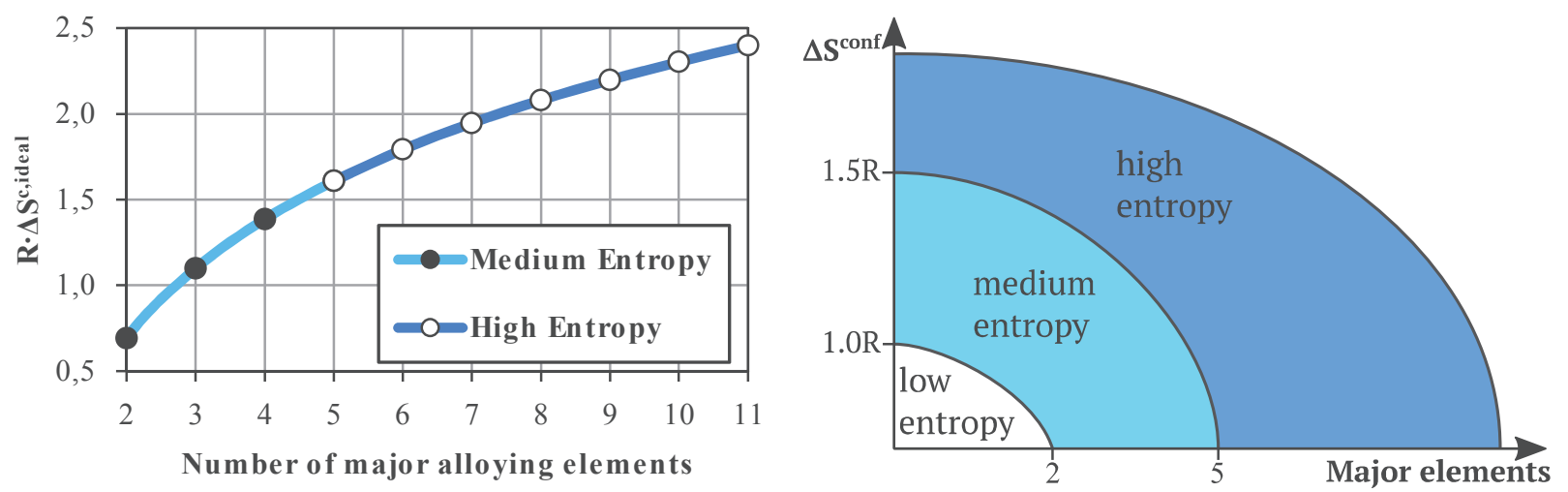

Figure 1 Schematic representation of early (left) and new (right) classifications of MPEA's

The nomenclature based on the foregoing definitions is most common in scientific literature and will be adopted by the authors for this work, however, there are other designations for MPEAs, among them: complex concentrated alloys (CCA), complex multicomponent alloys (CMA), compositionally complex alloys (CCA), baseless alloys (BA), etc. [15,16]. 
The primary definitions of HEAs and MEAs don't take into consideration any requirements that these alloys must contain only one or two solid solution phases but this, for a long time, was a main intended outcome in many researches considering the development of MPEAs. This was dictated by assumption that intermetallic phases can increase brittleness and promotion of simple solid solution structures should result in alloys possessing high strength while preserving considerable ductility [17]. However, over the years some new approaches have emerged, broadening the range of possibilities in the research and development of MPEAs even further than was initially thought. These approaches can be divided into three main categories [18]:

- $\quad$ Application based redesign - alloys are designed for specific applications.

- Enhancing the entropy of conventional alloys - existing alloys are modified with high entropy concepts.

- Incorporating other phases - formation of phases other than simple solid solutions is promoted.

Today the most research concerning MEA's and HEA's is done using samples produced by very rapid melt cooling or powder metallurgy [18]. Because the new routes in entropic alloys development do not exclude the possibility of formation of phases other than solid solution, however, there is potential to use slower cooling rates and thus conventional casting methods to produce functional parts made of these alloys. Using CALPHAD modelling Sanchez et. al. [19] designed a new group of lightweight and affordable casting MEA's. One of these alloys was selected to be investigated in this study.

\section{MATERIALSELECTION AND METHODOLOGY}

The alloy $\mathrm{Al}_{65} \mathrm{Cu}_{5} \mathrm{Cr}_{5} \mathrm{Si}_{15} \mathrm{Mn}_{5} \mathrm{Ti}_{5}$ used in this study was selected based on previous investigations presented in the work of Sanchez et al. [19]. Chemical composition of the alloy in at\% and wt\% is shown in Table 1.

Table 1 Chemical composition of selected alloy in weight and atomic percentages

\begin{tabular}{|c|c|c|c|c|c|c|}
\cline { 2 - 7 } \multicolumn{1}{c|}{} & Al & Cu & Si & Mn & Cr & Ti \\
\hline at\% & 65 & 5 & 15 & 5 & 5 & 5 \\
\hline wt\% & 53.7 & 9.7 & 12.9 & 8.4 & 8.0 & 7.3 \\
\hline
\end{tabular}

The alloys were manufactured in Balzers VSG-02 VIM furnace using high purity elemental ingredients, alumina crucible and protective atmosphere of Ar under pressure of $9 \cdot 10^{4} \mathrm{~Pa}$. The obtained ingot of $800 \mathrm{~g}$ was remelted three times under the same conditions to ensure its homogeneity. To control the melting and remelting temperature, a type S (Pt-PtRh) thermocouple in fused-silica tube was immersed periodically in molten metal, melt temperature was maintained at level of about $1200^{\circ} \mathrm{C}$.

The pattern for the test casting mould was designed to create three zones with different cooling rates (Figure 2). The first zone, a cube of dimensions $35 \times 35 \times 35 \mathrm{~mm}$, was designed to create cooling rate low enough to obtain accurate thermal-derivative readings. The thermal probe was installed in such a manner that the tip of type $S$ thermocouple, contained in protective fused silica glass tube, was in the area where the casting solidifies most slowly. The thermal-derivative analysis was carried out using Crystaldigraph 8T data recorder coupled with a laptop computer running ATDWin v1.3 thermal analysis software. The second zone of dimensions $20 \times 35 \times 10 \mathrm{~mm}$ was designed to create moderate cooling rate. The cooling rate was to be the highest in the last solidification zone consisting of a $10 \times 35 \times 5 \mathrm{~mm}$ channel connected to the brass plate embedded at the bottom of the mould.

The mould was made using typical $\mathrm{CO}_{2}$ process. The basic silica sand and bentonite clay mixture was manually rammed in steel flask around the pattern. After pattern removal the mould was purged by $\mathrm{CO}_{2}$ gas and left to dry and harden completely in vacuum dryer set to $150^{\circ} \mathrm{C}$ for 6 hours. 
The samples for DSC and structural analysis were cut out from second and third solidification zones. Exact sample cut is shown on Figure 2. Calorimetric analysis was done using Multi HTC S60 differential scanning calorimeter. Tests were carried out under Ar flow with the heating and cooling rate of $5^{\circ} \mathrm{C} / \mathrm{min}$, the temperature bracket for DSC measurement was set between 300 and $1200{ }^{\circ} \mathrm{C}$. Microstructure observation was conducted on Hitachi S-3400N SEM unit coupled with Thermo Noran EDS spectroscope using System Six software.

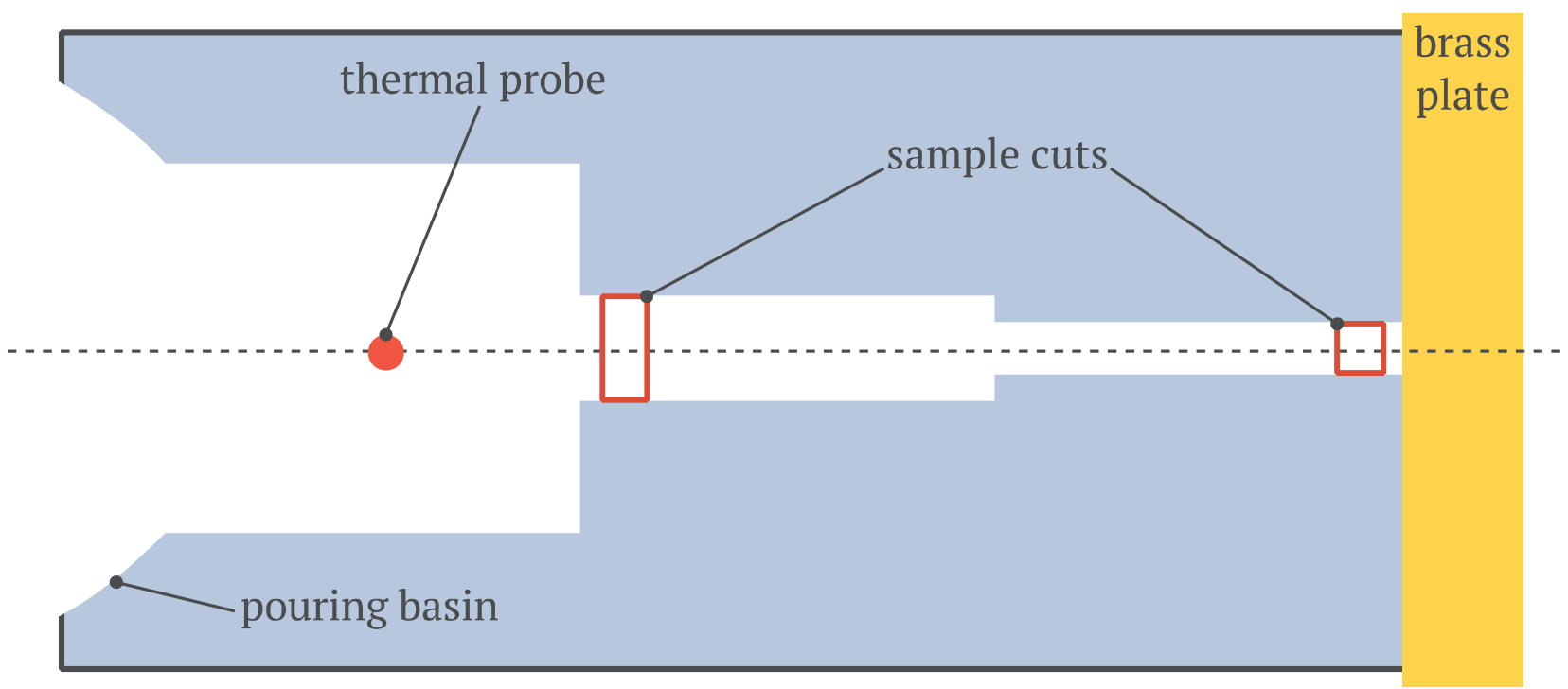

Figure 2 Schematic of mould channel with probe installation and sample extraction points

\section{RESULTS AND DISCUSSION}

The results of thermal-derivative analysis obtained during solidification of the test casting are presented in Figure 3. The pouring temperature of $1200{ }^{\circ} \mathrm{C}$ was insufficient to correctly register the liquidus temperature of the casting due to thermocouple inertia caused by the fused silica protective tube. Nevertheless, the analysis shows clearly as many as 6 thermal effects related to transformations during solidification. The overall solidification time was about 27 minutes. Investigated alloy has also extremely wide solidification range with the liquidus temperature above $1100{ }^{\circ} \mathrm{C}$ and solidus just below $500{ }^{\circ} \mathrm{C}$.

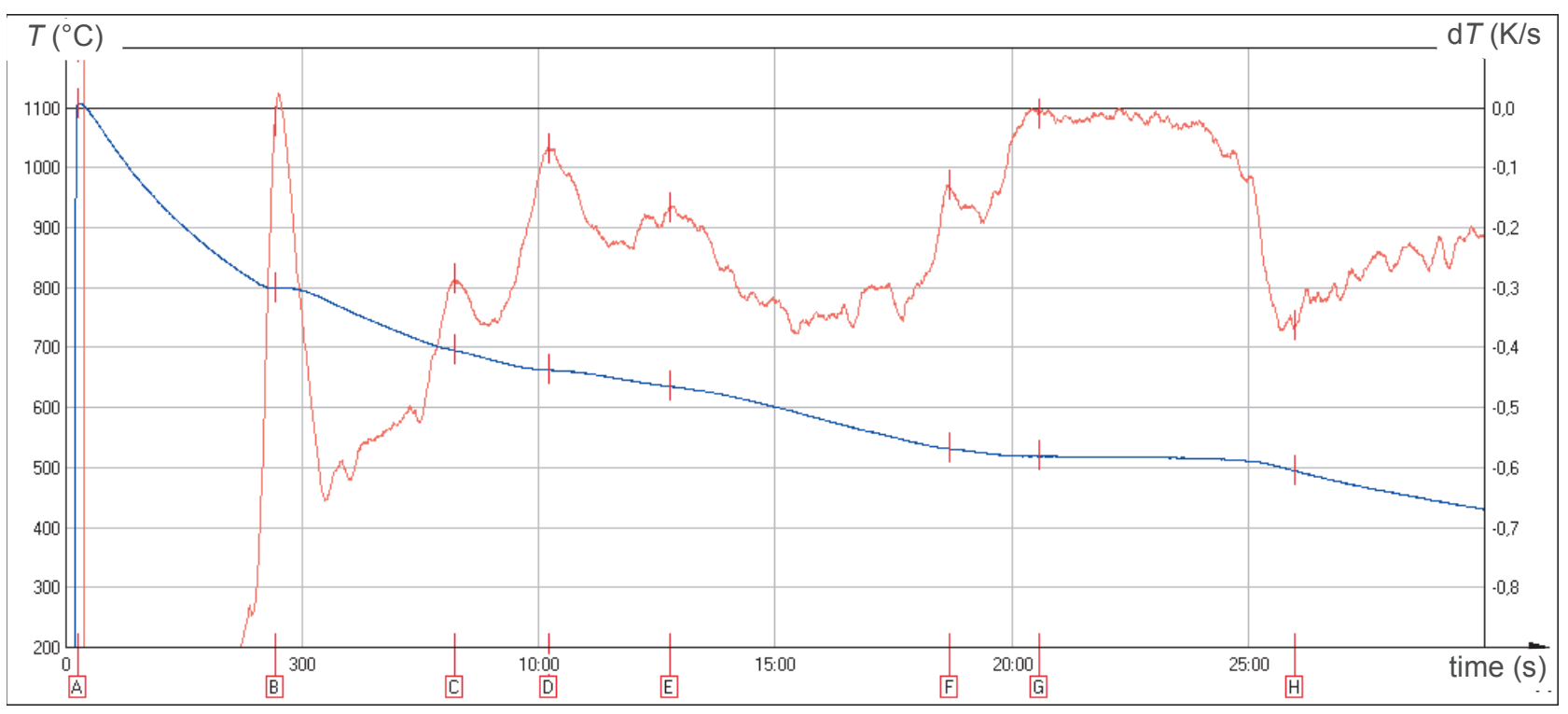

Figure 3 Thermal-derivative analysis graph with points indicating transformation temperature 

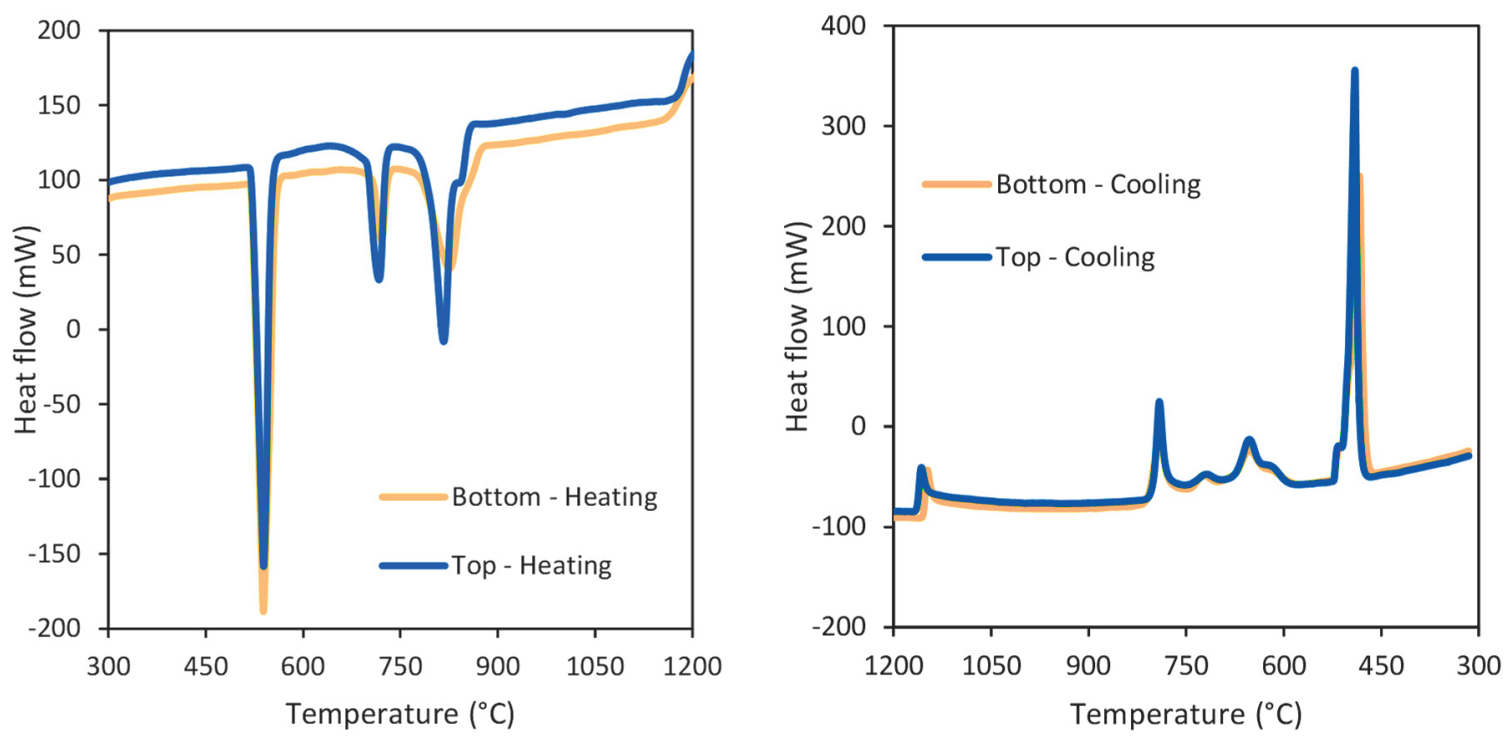

Figure 4 DSC curves for heating and cooling of the samples

The large number of transitions was confirmed by DSC measurements. DSC curves for both samples show roughly the same transition peaks during heating which indicate that the cooling rate difference between mould channels had not produced solidification conditions different enough to precipitate different phases. DSC data registered during cooling of the samples show the same 7 distinct transformations that were present on the thermal-derivative curves and the temperature of these transitions differs only slightly. DSC curves are presented in Figure 4. The bottom sample was cut out from the casting near the brass plate chiller and the top sample was cut out just below the thermal-derivative analysis cube as indicated on Figure 2.

Structural analysis shows large number of distinct phases corresponding to transitions registered by thermal analysis. Samples from different solidification rate zones are district only in the precipitation morphology and the size as shown on Figure $\mathbf{5}$.
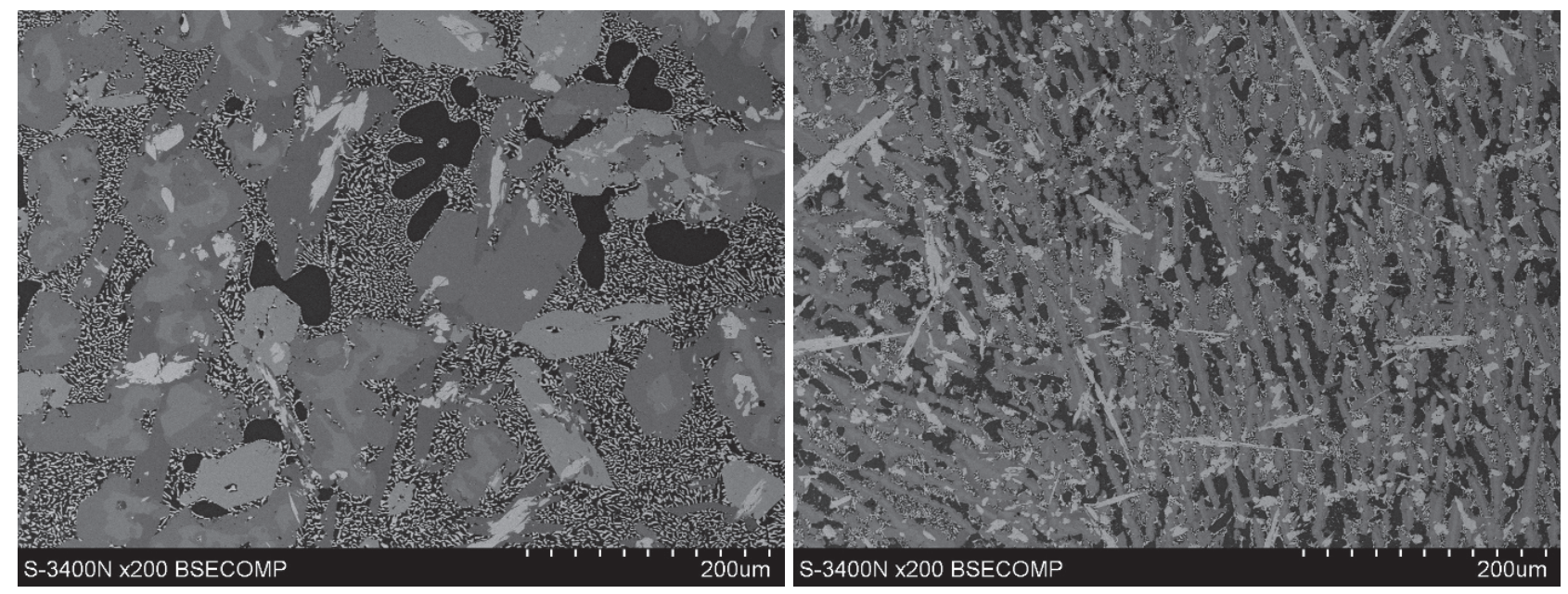

Figure 5 SEM images of microstructure of top sample (left) and bottom sample (right)

\section{CONCLUSIONS}

- Solid of selected alloy consists of conglomerate of as many as seven distinct phases precipitated out of liquid during solidification. 
- Solidification range of the alloy is extremely wide which excludes the possibility of its usage in casting.

- No significant differences between samples of different cooling rates were observed, excluding precipitation size and morphology.

\section{ACKNOWLEDGEMENTS}

\section{The work was funded by structural research BK-205/RM0/2019 (11/990/BK_19/0063).}

\section{REFERENCES}

[1] YEH, J.-W., CHEN, S.-K., LIN, S.-J., GAN, J.-Y., CHIN, T.-S., SHUN, T.-T., TSAU C.-H. and SHOU-YI, C. Nanostructured high-entropy alloys with multi-principal elements - novel alloy. Advanced Engineering Materials. 2004. vol. 6, no. 5, pp. 299-303.

[2] CANTOR, B., CHANG, I. T. H., KNIGHT P. and VINCENT, A. J. B. Microstructural development in equiatomic multicomponent alloys. Materials Science and Engineering A. 2004. vol. 375-377, no. 1, pp. 213-218.

[3] YEH, J.-W. Recent progress in high-entropy alloys. European Journal of Control. 2006. vol. 31, no. 6, pp. 633648.

TSAI, M.-H. and YEH, J.-W. High-entropy alloys: A critical review. Materials Research Letters. 2014. vol. 2, no. 3, pp. 107-123.

[4] RANGANATHAN, S. Alloyed pleasures: Multimetallic cocktails. Current Science. 2003. vol. 85, no. 10, pp. 14041406.

[5] TSAI, C., TSAI, M. H. and YEH, J.-W. Sluggish diffusion in Co-Cr-Fe-Mn-Ni high-entropy alloys. Acta Materialia. 2013. vol. 61, no. 13, pp. 4887-4897.

[6] ZHANG, Y., ZUO, T. T., TANG, Z., GAO, M. C., DAHMEN, K. A., LIAW P. K. and LU, Z. P. Microstructures and properties of high-entropy alloys. Progress in Materials Science. 2014. vol. 61, pp. 1-93.

[7] DIAO, H. Y., FENG, R., DAHMEN, K. A. and LIAW, P. K. Fundamental deformation behavior in high-entropy alloys: An overview. Current Opinion in Solid State and Materials Science. 2017. vol. 21, no. 5, pp. 252-266.

[8] MACKAY, A. L. On complexity. Crystallography Reports. 2001. vol. 46, no. 4, pp. 524-526.

[9] MURTY, B. S., YEH, J.-W. and RANGANATHAN, S. High-entropy Alloys. Boston: Butterworth-Heinemann, 2014.

[10] YEH, J.-W., CHEN, Y.-L., LIN S. J. and LEE, P.-H. High-entropy alloys - A new era of exploitation. Materials Science Forum. 2007. vol. 560, pp. 1-9.

[11] YEH, J.-W. Alloy design strategies and future trends in high-entropy alloys. JOM. 2013. vol. 65, no. 12, p. 17591771.

[12] GAO, M. C., YEH, J.-W., LIAW, P. K. and ZHANG, Y. High-Entropy Alloys - Fundamentals and Applications. Springer International Publishing, 2016.

[13] CAHN, R. W. and HAASEN, P. Physical Metallurgy. Amsterdam: North-Holland, vol. 1, 1996.

[14] MIRACLE, D. B. and SENKOV, O. A critical review of high entropy alloys and related concepts. Acta Materialia. 2017. vol. 122, pp. 448-511.

[15] ZHANG, W., LIAW, P. K. and ZHANG, Y. Science and technology in high-entropy alloys. Science China Materials. 2017. vol. 61, no. 1, pp. 2-22.

[16] MIRACLE, D. B. Critical assessment 14: High entropy alloys and their development as structural materials. Materials Science and Technology. 2015. vol. 31, no. 10, pp. 1142-1147.

[17] TSAI, M. H. Three strategies for the design of advanced high-entropy alloys. Entropy. 2016. vol. 18, pp. 1-14.

[18] SANCHEZ, J. M., VICARIO, I., ALBIZURI, J., GURAYA, T. and ALONSO, J. C. G. Phase prediction, microstructure and high hardness of novel light-weight high entropy alloys. Journal of Materials Research and Technology. 2019. Vol. 8, no. 1, pp. 1-1592. DOI: 10.1016/j.jmrt.2018.06.010. 